\title{
Estimation and prevention of text neck syndrome among smart phone users
}

\author{
Kamalakannan M. ${ }^{1}$, Rakshana R. ${ }^{2}$, Padma priya R. ${ }^{2}$ \\ ${ }^{1}$ Assistant Professor, ${ }^{2}$ Physiotherapy Undergraduate, Saveetha College of Physiotherapy, SIMATS, Chennai, Tamil Nadu, \\ India
}

(Received: August $2019 \quad$ Revised: June $2020 \quad$ Accepted: August 2020)

Corresponding author: Kamalakannan M. Email: kamal1712@gmail.com

\begin{abstract}
Introduction and Aim: Text neck syndrome has become a global musculoskeletal problem in relation to all the ages who uses the mobile phone. The aim of the study was to investigate the neck posture, self-report of pain and disability in smart phone users, and to identify the preventive measures of text neck syndrome.
\end{abstract}

Materials and methods: 253 students were selected according to the inclusion and exclusion criteria. They were assessed by measuring the resting head posture using a ruler's method and A self-administered questionnaire was distributed to all subjects. The data obtained was tabulated and statistically analysed.

Results: Results were statistically analysed using Chi-square test. Questionnaire includes totally 10 domains. Each question is given with three to five options.

Conclusion: Frome the study it was concluded that most of the people are using phone in the non- ergonomic way. $90 \%$ of the people were affected by neck related musculoskeletal problems. Prevention is the only key to avoid text neck syndrome.

Keywords: Mobile phone; neck posture; cervical spine; text neck syndrome; hazards; preventive measures.

\section{INTRODUCTION}

$\mathrm{T}$ ext neck syndrome has become a global epidemic affecting the larger number of population of almost all the ages who uses the mobile phone. In olden days, the usage of phone is only for voice and data communication, but current mobile phone is used for many purposes like messaging, gaming, face book and other educational and entertainment purposes (1). "Text neck" was a term coined by Dr. Dean Fishman, who is a US Chiropractor. The term text neck or another phrase "turtle neck posture" can be described as overuse syndrome or repetitive stress injury to the neck caused by having head in forward and downward position looking at his or her phone or other electronic devices for extended period of time (2). Text neck syndrome may cause harmful effects on cervical spine, supporting musculature, ligaments, nerves, tendons and bony segments eventually reversing the natural curvature of spine, leading to early spinal arthritis, disc degeneration, headache, up to $30 \%$ decrease in lung capacity (3). In anatomical posture the line of gravity passes through the external auditory meatus, the bodies of cervical spine and acromion and anterior to thoracic spine. Undue stress is imposed on the vertebrae of lower neck due to forward pull of the weight of the head leads to degeneration of disk and other degenerative neck problems. Poor posture can cause the upper back muscle to work continuously to counterbalance the pull of the gravity and forward neck (4).

The neck or cervical spine is a coordinated network of bones, muscles, joints, nerves directed by the brain and spinal cord. Irritation of nerve leads to pain along the pathway of distribution of nerve. Recent study found that $79 \%$ of people use their cell phones with them almost all the time. Text neck syndrome directly affects the spine by flexing the head forward at varying degree. When the head tilts forward at 15 degree, it is sufficient to produce 27 pounds; at 30 degree it produces 40 pounds of force; at 45 degree it produces 49 pounds of force; at 60 degree it produces 60 pounds of force; then at 90 degree the model prediction was not reliable. This problem is one of the major concern in children because the size of their head is too large in relation to the body size than adults, thus they are at higher risk of text neck (5). In upright posture, the ears are aligned with the centre of the shoulders; hence the average weight experienced by the head is approximately six times as much same weight as an average 8years old baby (6).

Poor posture leads to fatigue, which will have a negative effect like reduced physiological function, disruption of automatic nervous system and leads to creation of problem in daily life. Musculoskeletal disorder in relation to smartphone use includes fatigue of muscles and increased loading of neck muscles. As a result pain, stiffness, insensitivity to pain in neck and shoulder may appear (7). Some of the common symptoms of text neck syndrome are, Upper back pain or neck pain when using handheld device, Nagging or sharp pain in the neck or shoulders at the end of the day, General shoulder pain and tightness, Intermittent or constant headache made worse when looking down or using computer, If a cervical nerve becomes pinched, neurological symptoms can radiate down to arm and into hand. If left untreated, it leads to severe inflammation of neck 
ligaments, muscles, nerves and arthritic damage and leads to permanent serious damage such as flattening of spinal curve, onset of early arthritis, spinal malalignment, spinal degeneration, disc compression, nerve damage, GI problems, loss of lung volume and capacity, stiff neck, radiating pain and headache $(8$, 9).

Precautions from developing sickness must be taken. Proper handling of smart phone, simple postural correction exercise, neck stabilisation exercise, and soft tissue massage can help to decrease the development of cervical and upper extremity musculoskeletal disorder. Global awareness need to be spread among public and medical community (10). Some of the techniques done to get relief from text neck are mentioned below: Warm up your neck muscles time to time, stretches, chin and scapula retraction, rest, talk more and text less, apply ice or heat, massage, adapt better posture, and modify lifestyle (11).

\section{METHODOLOGY}

The study design was an observational study with a sample size of 253. Study was conducted for students of Saveetha College of physiotherapy. Ethical clearance was obtained from institutional review board. 19 to 21 years of age group were included for the study. They were assessed by measuring the resting head posture using a ruler's method, subjects were asked to obtain a relaxed posture with their scapula touching against the wall and then we used an inch tape to measure how far the back of the head is away from the wall. If the head touches the wall while standing naturally up straight, there is no forward head posture. Subjects who are at the higher risk of getting text neck syndrome are included in the study based on ruler's method. Exclusion criteria were subjects having any congenital cervical problems and subjects with traumatic and pathological cervical problems.

Questionnaire: self-administered questionnaire was given to all participants to collect information related to usage of phone, knowledge about text neck syndrome, health hazards of excess phone usage.

Exercises, which can prevent text neck syndrome, are Nod of head; Chest opening and Spinal decompression/postural correction by following these exercise daily $10 \mathrm{Rep} / 3$ sets daily can prevent the severity of text neck syndrome.
If needed hot pack can be administered 2 times daily and use of proper pillow can further help to reduce the symptoms condition.

\section{Statistical analysis}

Statistical analysis was done on a blinded, intention to treat basis using SPSS software (version 18.0). Chi-square test were conducted to compare the statistical data's of the study, $\mathrm{P}<0.05$ was set for all analyses and the significance level was set to $\alpha=$ 0.05 .

\section{RESULTS}

Results were statistically analysed using chi-square test. Questionnaire includes totally 10 domains. Each question is given with three to five options. In the domain one $60 \%$ of the subjects were using moderate weight, $30 \%$ using light weight and $10 \%$ using heavy weight. In the second domain $70 \%$ of the subjects using mobile phone for more than 5hours, $20 \%$ using phone for around 2hours, $10 \%$ using less than 2hours. In the third domain $65 \%$ of the subjects using phone at the level of waist, $20 \%$ using at the level of hip, $15 \%$ using at the level of hip. In the fourth domain $75 \%$ of the subjects using phone in flexed position of the neck, $15 \%$ using phone in neutral position of the neck and remaining $10 \%$ using phone in neck extended position. In the fifth domain $85 \%$ of the subjects doesn't feel headache while using phone, $7.5 \%$ have mild headache, $5 \%$ have moderate headache, $2.5 \%$ have severe headache. In the sixth domain around $75 \%$ of the subjects using phone for nearly about 2 to 4 years, $15 \%$ using phone above 4 years, $7.5 \%$ using phone for the past 1 to 2 years, $2.5 \%$ using phone for less than 1 year. In the seventh domain $80 \%$ of the subjects use sitting position while using phone, $12.5 \%$ using in supine lying, $5 \%$ using phone in side lying, $2.5 \%$ using in prone lying. In the eight domain $70 \%$ of the subjects complains of pain while looking down, $20 \%$ while looking up, 5\% each while tilting head to right and left side. In the ninth domain $72.5 \%$ of the subjects have very mild pain while using the phone, $12.5 \%$ of the subjects have moderate pain, $7.5 \%$ have severe pain, and $7.5 \%$ have no pain. In the tenth domain $90 \%$ of the population is aware of the term "Text neck syndrome", $10 \%$ is unaware of text neck syndrome. 
Kamalakannan et al: Estimation and prevention of...... phone users

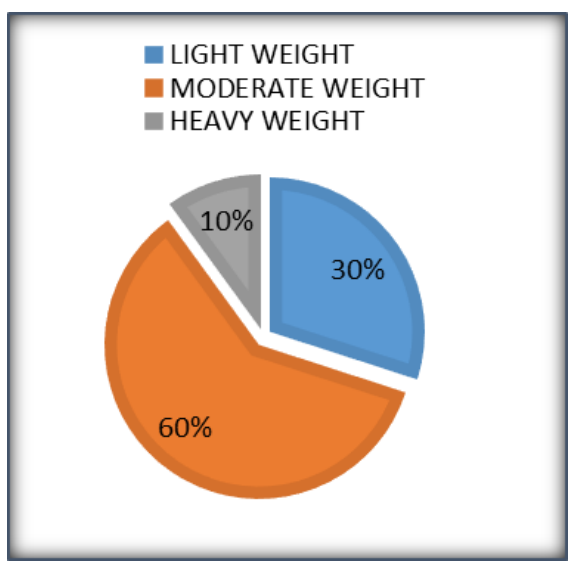

Fig. 1: Domain 1-Phone weight

a EYE LEVEL $\square$ WAIST LEVEL $\square$ HIP LEVEL

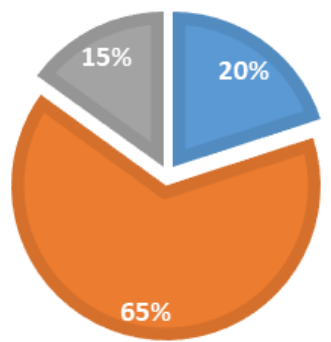

Fig. 3: Domain 3-Phone holding a position

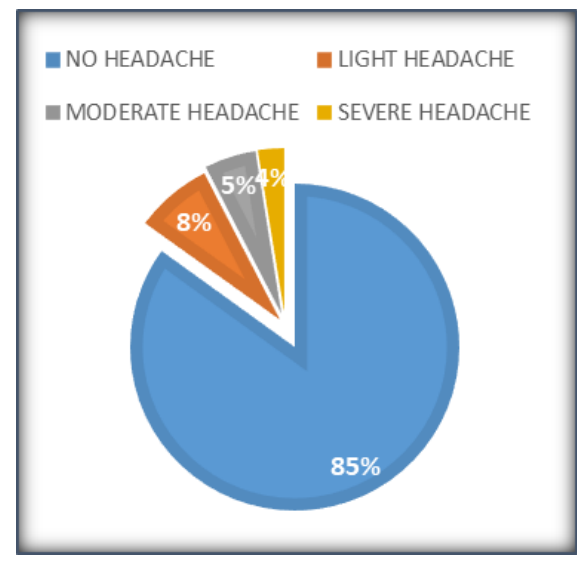

Fig. 5: Domain 5-Headache while using phone

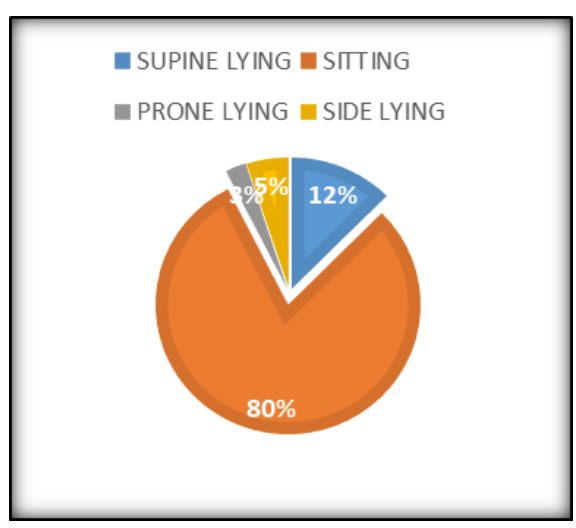

Fig. 7: Domain 7-Phone using position

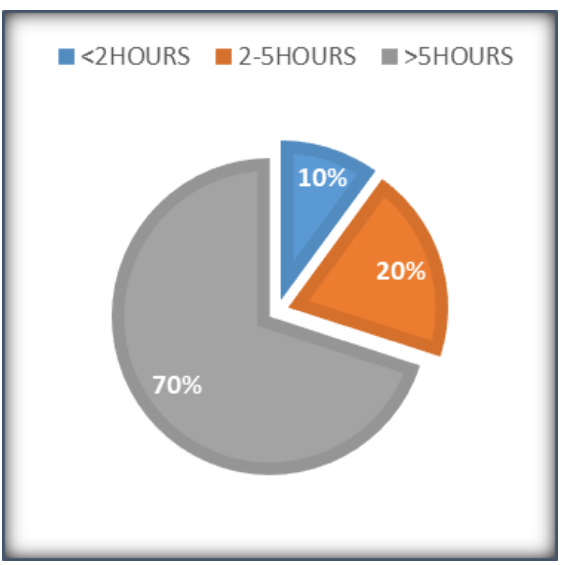

Fig. 2: Domain 2-Phone usage hours

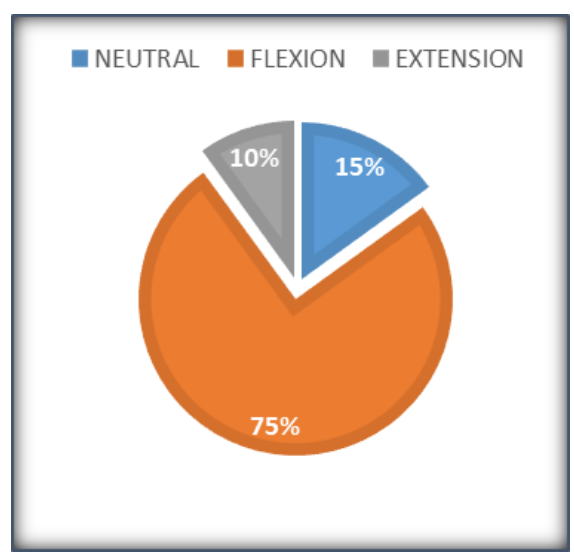

Fig. 4: Domain 4-Neck position while using Phone

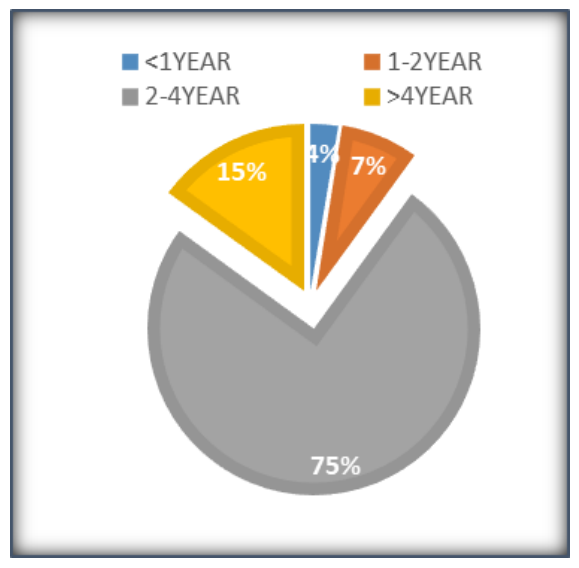

Fig. 6: Domain 6-6 years of using phone

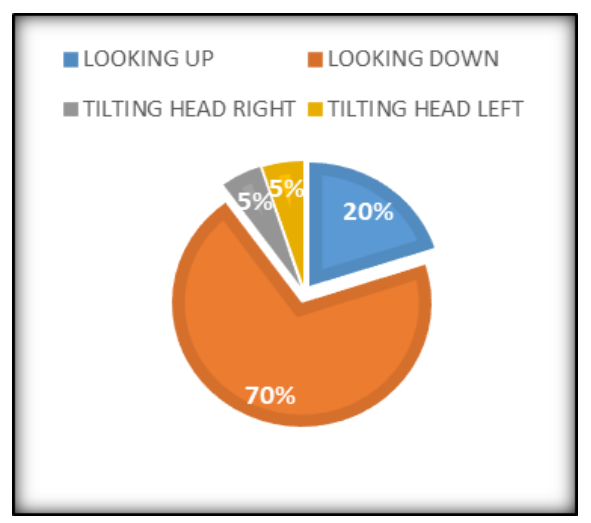

Fig. 8: Domain 8-Position that induces pain 


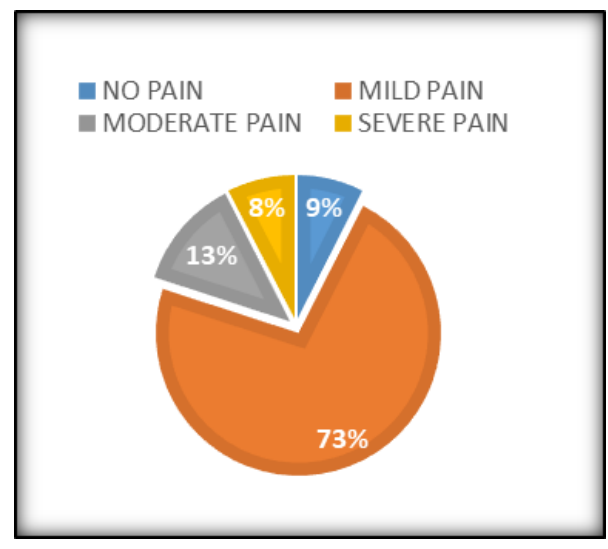

Fig. 9: Domain 9-Pain intensity

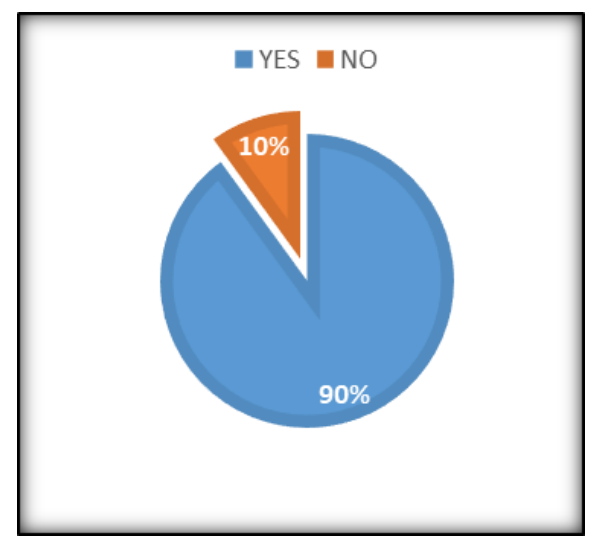

Fig. 10: Domain 10-Awareness of text neck syndrome

\section{DISCUSSION}

Texting on the smart phone is an activity, which requires looking into mobile screens in a flexed position of the neck with a forward head posture and with a rounding of shoulder and movement of the thumb and arms in a continuous pattern. When a person maintains this position for extended period of time, then head is pushed forward. This imbalance is counterbalanced by constant contraction of musculature of neck giving rise to text neck syndrome with symptoms such as neck pain, shoulder pain, upper back pain, forward head posture, and muscle spasm (12). In this study 19 to 21 years of age group were included since mobile phone usage where increased between college going students.

People are using their phone during walking, sitting and also in lying on prone and side lying. The posture which the people adopt to look at their smart phone increases the stress on the neck. Further, people are tilting their head while using the phone due to the size of the equipment. It may cause excessive stress on the related structures (13-15).

In a recent study published last year in Clinical Psychology and Psychotherapy of people with clinical depression, subjects were randomly selected to sit in either a slouched or an upright position and they were presented with a list of positive and negative words. The slouches showed a negative recall bias when they were asked to recall later (remembering the bad stuff more than the good stuff), while those who sat upright showed no such bias. Thus people adopting slouched position while using phone, it directly affects the memory is proved (1618).

Another study in Sweden focused on whether there is any relation between texting on mobile phone and musculoskeletal disorder in the neck and upper extremities in a population of young adults. This study found that there is an association between text messaging and reported ongoing symptoms in neck

and upper extremities. (19-21). another study conducted by Hansraj states that weight of the head normally ranges between 10-20lb. But weight on the neck increases while flexion of the cervical spine increases during texting, the maximum weight being $60 \mathrm{lb}$ at 60 degrees (22). Recent research has shown that smartphone usage can cause severe soft tissue strain and pain in the shoulders, neck, and thumbs. Prolong usage of smartphone can lead to rounded shoulders.

\section{CONCLUSION}

$90 \%$ of the people were affected by neck related musculoskeletal problems by using mobile phone in non-ergonomic way. $90 \%$ of the people were affected by neck related musculoskeletal problems. Prevention is the only key to avoid text neck syndrome. Certain important things should be incorporated in our daily life to prevent text neck syndrome. They are: avoid prolonged usage of phone, take adequate rest time, avoid excessive static posture, use the device in a position such that it reduces the stress on head, neck and upper extremity, avoid excessive repetition of movements such as typing or swiping, avoid holding heavy weight phone or any other devices in one hand for longer period of time.

\section{CONFLICT OF INTEREST}

There is no conflict of interest from other authors.

\section{REFERENCES}

1. Kumar, L. R., Chii, K. D., Way, L. C., Jetly, Y., Rajendaran, V. Awareness of mobile phone hazards among university students in a Malaysian medical school. Health. 2011; 3(07): 406.

2. Neupane, S., Ali, U. I., Mathew, A. Text neck syndromeSystematic review. Imperial J Interdisciplinary Res. 2017; 3(7): 141-148.

3. Vate, Lan P., Text neck epidemic: a growing problem for smart phone users in Thailand. Int $\mathrm{J}$ Comput Internet Manag. 2015; 23(3): 1-55.

4. Singla, D., Vegar, Z. Association between forward head, rounded shoulders and increased thoracic kyphosis: A reviewed of the literature. Pubmed. 2017; 16(3): 220-229.

5. Gharbawi, B. Text Neck Syndrome: A new concern for physical therapists worldwide. EC Orthopaedics. 2017; 8(3): 89-91. 
6. Kim, H. J., Kim, J. S. The relationship between smartphone use and subjective musculoskeletal symptoms and university students. J Physical Therapy Sci. 2015; 27(3): 575-579.

7. Lee, J. I., Song, H. S. The correlation analysis between hours of smartphone use and neck pain in the Gachon university students. The Acupuncture. 2014; 31(2): 99-109.

8. Lee, H. J. Neck and functioning in daily activities associated with smartphone usage. Jkpt. 2016; 28(3): 183-188.

9. Kim, H. J., Kim, J. S. The relationship between smartphone use and subjective musculoskeletal symptoms and university students. J Phys Ther Sci. 2015; 27(3): 575-579.

10. Xie, Y., Szeto, G., Dai, J. Prevalence and risk factors associated with musculoskeletal complaints among users of mobile handheld devices: A systematic review. Applied Ergonomics. 2017; 59: 132-142.

11. AlZarea, B. K., Patil, S. R. Mobile phone head and neck pain syndrome: proposal of a new entity. OHDM Headache. 2015; 14(5): 313-317.

12. Kim, M. S. Influence of neck pain on cervical movement in the sagittal plane during smartphone use. J Physical Therapy Sci. 2015; 27(1): 15-17.

13. Hansraj, K. K. Assessment of stresses in the cervical spine caused by posture and position of the head. Surg Technol Int. 2014; 25: 277-279.

14. Gustafson, E., Thomee, S., Grimby-Ekman, A., Hagberg, M. Texting on mobile phones and musculoskeletal disorders in young adults: a five-year cohort study. Applied Ergonomics. 2017; 58: 208-214.

15. Kamalakannan, M., Anitha, A. Effectiveness of lower trapezius exercise in pain and disability among chronic neck pain subjects. International Journal of Therapies and Rehabilitation Research. 2015; 4(5): 283-286.

16. Malik, A., Pasha, M. U., Khalid, S., Ahmad, A., Gilani, S. A. Prevalence of neck pain among Undergraduate students of Lahore. Int J Scientific Engineering Res. 2017; 8(10): 569-576.

17. Damasceno, G. M., Ferreira, A. S., Nogueira, L. A. C. Text neck and neck pain in 18-21-year-old young adults. Eur Spine J 2018; 27: 1249-1254.

18. Kamalakannan, M., Sangeetha Valli, M., Nivetha, S., Augustina, J. A study to compare the sagittal posture analysis for single sided and double-sided backpack users. Biomedicine. 2019; 39(4): 628-632.

19. Kalaiselvan, A., Kamalakannan, M., Sowmya. Effect of backpack of $10 \%$ of the body weight on cervical and shoulder posture for school going children. International Journal of Pharma and Biosciences. 2016; 7(4): 125-129.

20. Kamalakannan, M., Kalyani. Effectiveness of dynamic neck exercises along with interferential therapy in patients with chronic mechanical neck pain. International Journal of Academic Research and Development. 2018; 3(2): 927-932.

21. Sharmila, B., Alagesan. J. Pain and disability in secondary school teachers- Correlation study. International Journal of Pharma and Biosciences. 2015; 6(4): 254-257.

22. Misra, A., Nigam, M., Alagesan, J. Effect of exercises in cervical postural deviation due to backpack in school children. International Journal of Current Research. 2012; 4(8): 146-149. 